ARTICLE

https://doi.org/10.1038/s41467-019-13944-2

\title{
Edge stabilization in reduced-dimensional perovskites
}

Li Na Quan (10 1,12, Dongxin Ma (D) 1,12, Yongbiao Zhao 1,2,12, Oleksandr Voznyy (1) 1, Haifeng Yuan (D) 1,3, Eva Bladt ${ }^{4}$, Jun Pan (1) 5,11, F. Pelayo García de Arquer (10 1, Randy Sabatini ${ }^{1}$, Zachary Piontkowski ${ }^{6}$, Abdul-Hamid Emwas ${ }^{7}$, Petar Todorović1, Rafael Quintero-Bermudez1, Grant Walters', James Z. Fan', Mengxia Liu (1) 1, Hairen Tan (1) 1, Makhsud I. Saidaminov ${ }^{1}$, Liang Gao ${ }^{1,8}$, Yiying $\mathrm{Li}^{2}$, Dalaver H. Anjum7, Nini Wei ${ }^{7}$, Jiang Tang ${ }^{8}$, David W. McCamant ${ }^{6}$, Maarten B.J. Roeffaers ${ }^{9}$, Sara Bals (1) ${ }^{4}$, Johan Hofkens ${ }^{3,10}$, Osman M. Bakr ${ }^{5}$, Zheng-Hong Lu (i) ${ }^{2 \star} \&$ Edward H. Sargent (1) ${ }^{1 \star}$

Reduced-dimensional perovskites are attractive light-emitting materials due to their efficient luminescence, color purity, tunable bandgap, and structural diversity. A major limitation in perovskite light-emitting diodes is their limited operational stability. Here we demonstrate that rapid photodegradation arises from edge-initiated photooxidation, wherein oxidative attack is powered by photogenerated and electrically-injected carriers that diffuse to the nanoplatelet edges and produce superoxide. We report an edge-stabilization strategy wherein phosphine oxides passivate unsaturated lead sites during perovskite crystallization. With this approach, we synthesize reduced-dimensional perovskites that exhibit $97 \pm 3 \%$ photoluminescence quantum yields and stabilities that exceed $300 \mathrm{~h}$ upon continuous illumination in an air ambient. We achieve green-emitting devices with a peak external quantum efficiency (EQE) of $14 \%$ at $1000 \mathrm{~cd} \mathrm{~m}^{-2}$; their maximum luminance is $4.5 \times 10^{4} \mathrm{~cd} \mathrm{~m}^{-2}$ (corresponding to an EQE of $5 \%$ ); and, at $4000 \mathrm{~cd} \mathrm{~m}^{-2}$, they achieve an operational halflifetime of $3.5 \mathrm{~h}$.

\footnotetext{
${ }^{1}$ Department of Electrical and Computer Engineering, University of Toronto, 10 King's College Road, Toronto, ON M5S 3G4, Canada. ${ }^{2}$ Department of Materials Science and Engineering, University of Toronto, 184 College Street, Toronto, ON M5S 3E4, Canada. ${ }^{3}$ Department of Chemistry, KU Leuven, Celestijnenlaan 200F, B-3001 Leuven, Belgium. ${ }^{4}$ EMAT, University of Antwerp, Groenenborgerlaan 171, 2020 Antwerp, Belgium. ${ }^{5}$ Division of Physical Science and Engineering, King Abdullah University of Science and Technology (KAUST), Thuwal 23955-6900, Saudi Arabia. ${ }^{6}$ Department of Chemistry, University of Rochester, 120 Trustee Rd., Rochester, NY NY14627, USA. ${ }^{7}$ Core Labs, King Abdullah University of Science and Technology, Thuwal 239556900, Saudi Arabia. ${ }^{8}$ Wuhan National Laboratory for Optoelectronics (WNLO), Huazhong University of Science and Technology (HUST), 430074

Wuhan, China. ${ }^{9}$ Centre for Surface Chemistry and Catalysis, KU Leuven, Celestijnenlaan 200F, B-3001 Leuven, Belgium. ${ }^{10}$ Max Planck Institute for Polymer Research, Ackermannweg 10, Mainz 55128, Germany. "11Present address: College of Materials of Science and Engineering, Zhejiang University of Technology, Hangzhou, China. ${ }^{12}$ These authors contributed equally: Li Na Quan, Dongxin Ma, Yongbiao Zhao. *email: zhenghong.lu@utoronto.ca; ted.sargent@utoronto.ca
} 
educed-dimensional metal halide perovskites (MHPs) are an emerging class of materials that hold advantages in optoelectronics relative to conventional three-dimensional (3D) $\mathrm{MHPs}^{1-7}$. Reduced-dimensional MHPs are intermediate between 3D and two-dimensional (2D) perovskites: they are synthesized via control over the concentration of large organic cations incorporated in between perovskite layers. The added organic cations confining perovskite layers increase the formation energy and mitigate chemical degradation in the presence of moisture ${ }^{8,9}$, enabling solar cells exhibiting improved stability compared to their $3 \mathrm{D}$ counterparts ${ }^{10-12}$. Strong and tunable confinement allows the exciton binding energy to be increased well above the thermal dissociation threshold, enabling increased radiative rates for light-emission applications ${ }^{13-16}$. The multiple quantum wells of varying thicknesses provide cascade energy transfer among domains with different bandgaps, leading to photoluminescence quantum yields (PLQYs) of over $60 \%$ at low pump power densities ${ }^{17}$.

However, reduced-dimensional MHPs still show limited stability under sustained photoexcitation and electrical injection, and this remains a roadblock to their deployment in lightemitting diodes (LEDs) ${ }^{17,18}$. Understanding of the mechanisms behind this degradation have benefited from a number of important studies: it was shown that long-lived free carriers accumulate at the edge of reduced-dimensional MHPs, leading to a high density of dangling bonds and unsaturated atoms. The edge states in reduced-dimensional MHPs refer to the states that are chemically unstable, structurally uncovered by organic amines. These exciton-accepting edge states are susceptible to moisture and oxygen, and under photoexcitation they are the recipients of significant carrier transfer, especially in widebandgap materials ${ }^{19}$.

Here we investigate the degradation mechanism in reduceddimensional MHPs using a combined computational and experimental strategy. We study the role of these sites in photodegradation and then devise an edge-stabilization strategy to mitigate this problem. This enables us to report the longest device operational lifetime at high luminance $\left(4000 \mathrm{~cd} \mathrm{~m}^{-2}\right)$, by a margin of $>21$ times, relative to the best prior report (at the initial luminance of $3800 \mathrm{~cd} \mathrm{~m}^{-2}$, with $\left.T_{50}=10 \mathrm{~min}\right)^{20}$.

\section{Results}

Structural analysis of reduced-dimensional perovskites. We focused on reduced-dimensional MHPs with a stoichiometry of $\mathrm{PEA}_{2} \mathrm{Cs}_{2.4} \mathrm{MA}_{0.6} \mathrm{~Pb}_{4} \mathrm{Br}_{13}$ (here PEA is phenylethylammonium and $\mathrm{MA}$ is methylammonium). We synthesized the perovskites using a one-step spin-coating method. The films showed green emission peaked at $517 \mathrm{~nm}$ and exhibited a high PLQY of $60 \%$. The optimization of the Cs-to-MA ratio revealed that an appropriate amount of MA was important to achieve high PLQYs (Supplementary Table 1$)^{18}$.

We obtained the nanoplatelet thickness distribution required for energy funneling. We used aberration-corrected low-dose high-angle annular dark field (HAADF) scanning transmission electron microscopy (STEM) (Fig. 1 and Supplementary Fig. 1). Individual sheets consisting of two to four $\mathrm{PbBr}_{6}$ unit cells were clearly resolved. The distance between stacked sheets was $1.5-1.6 \mathrm{~nm}$, corresponding to the PEA organic interlayer thickness. Multiple step edges were also resolved in STEM images (Supplementary Fig. 2), but could not exclude that such a step would be induced by the interaction of the highly energetic electrons with the perovskite outer surface.

Conceptual design of edge-stabilization strategy. Previous work on $3 \mathrm{D}$ perovskite solar cells ${ }^{21}$ has shown that, when MHPs are photoexcited, the surface-localized excitons or carriers transfer to the adsorbed oxygen molecules, turning them into superoxide $\left(\mathrm{O}_{2}{ }^{-}\right)$that triggers perovskite oxidation and decomposition. A photodegradation pathway is triggered when an electron is transferred from the perovskite to $\mathrm{O}_{2}$, creating a superoxide $\left(\mathrm{O}_{2}{ }^{-}\right)$that irreversibly splits and converts into a chemisorbed oxide species.

Density functional theory (DFT) calculations indicated that the unsaturated $\mathrm{Pb}$ dangling bonds do not, on their own, form trap states (the unsaturated $\mathrm{Pb}$ dangling bonds were exposed due to the loss of $\mathrm{PEA}^{+}$capping ligand or PEABr) (Fig. 2a and Supplementary Figs. 3-7) $22-24$. However, they remain susceptible to the adsorption of a variety of nucleophilic molecules (e.g. oxygen molecules) that readily forms a dative bond with the surface. The physically adsorbed oxygen molecules result in
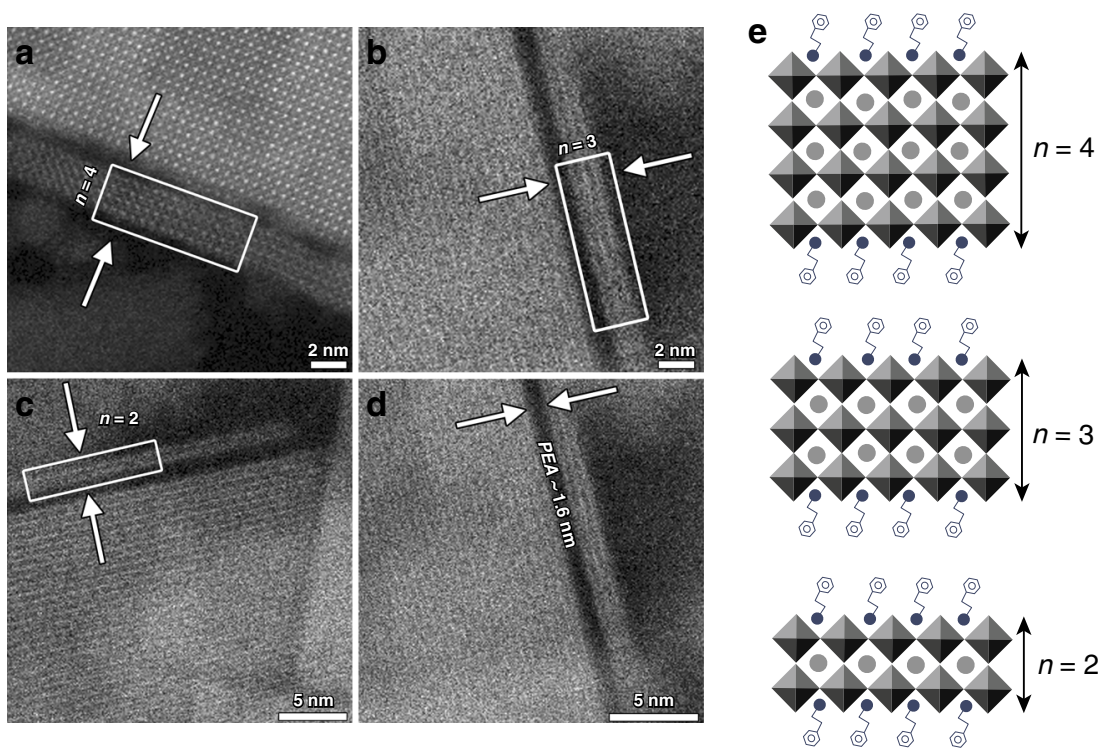

Fig. 1 Visualization of reduced-dimensional perovskites. High-angle annular dark field (HAADF) scanning transmission electron microscopy (STEM) images of the layered perovskites exhibiting domains with different number of layers (a-d), where in $\mathbf{a}$, a four-layered structure was observed. 
a
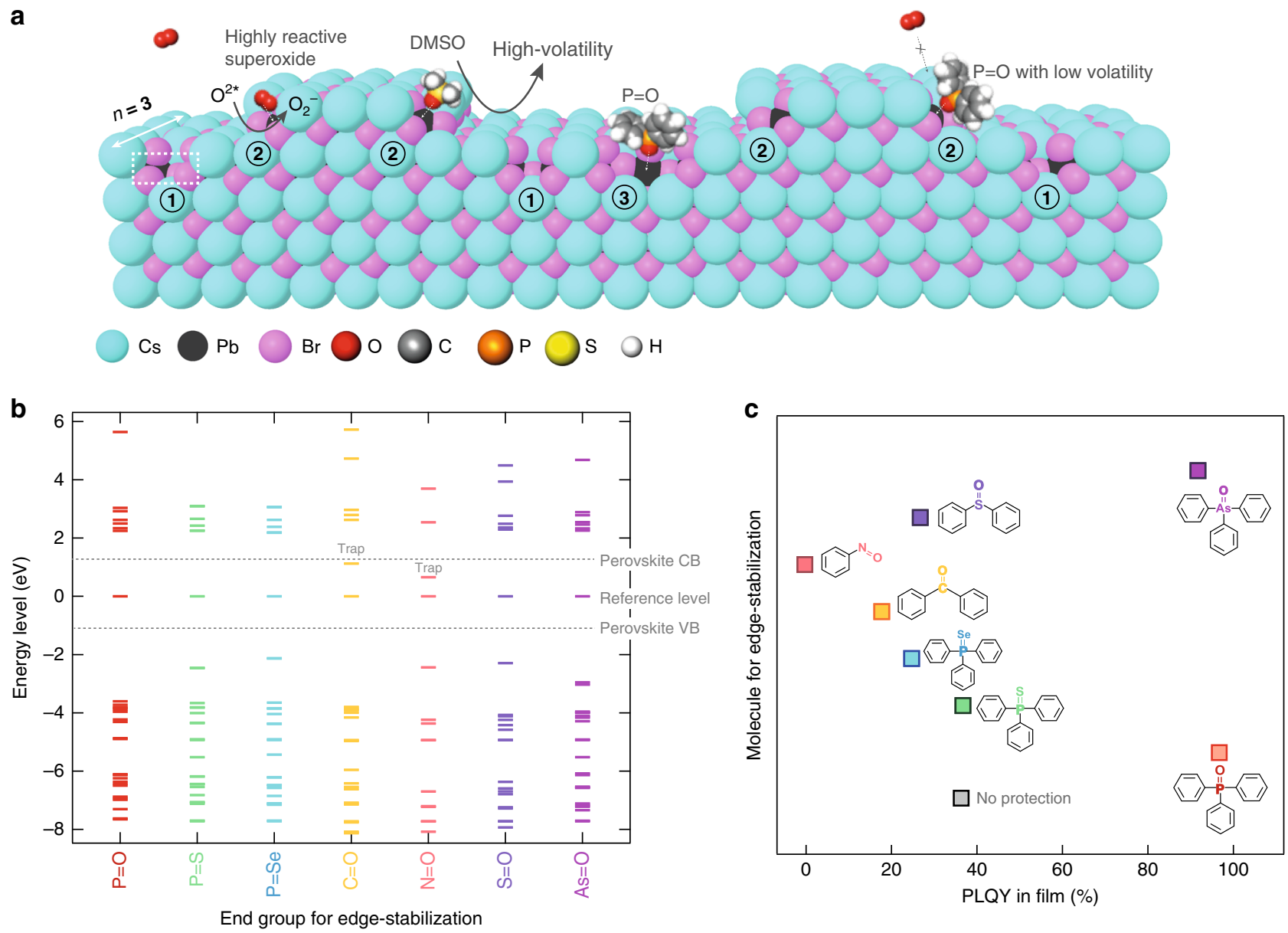

Fig. 2 Photo-induced degradation mechanisms and edge-stabilization strategy. a Schematic illustrating imperfect edges in $n=3$ reduced-dimensional perovskites of $\mathrm{PEA}_{2} \mathrm{Cs}_{n-1} \mathrm{~Pb}_{n} \mathrm{Br}_{3 n+1}$ and proposed reaction pathway of superoxide production under photoexcitation, including (1) missing Cs ${ }^{+}$or $\mathrm{PEA}^{+}$at the edge sites, (2) missing $\mathrm{Cs}^{+}$or $\mathrm{PEA}^{+}$at the corner sites, (3) desorbed $\mathrm{CsBr}$ or PEABr near the defect. $\mathbf{b}$ Energy level alignment obtained from DFT calculations. c PLQYs of the perovskites treated using different molecules.

electronic traps in the perovskite bandgap, a phenomenon also seen in other semiconductors (Supplementary Fig. 7) ${ }^{25,26}$.

We hypothesized that introducing a benign Lewis base adduct - one that outcompeted oxygen adsorption-could improve the stability of perovskites in an oxygen-rich environment. Typical Lewis base polar aprotic solvents have been applied to dissolve perovskite precursors, such as dimethyl sulfoxide (DMSO), dimethyl formamide (DMF) and $N$-methyl-2-pyrrolidone (NMP), or form adducts with the metal halides, and therefore are widely used to impede the fast formation of perovskite crystals and to control film morphology $27-29$. However, these Lewis base-metal complexes formed with volatile solvents failed to withstand the annealing step during film fabrication (Fig. 2a). Reliance on this approach therefore left metal dangling bonds exposed to oxygen attack ${ }^{30}$.

We sought the materials that would combine the desired electronic and edge-stabilizing properties, and that would be sufficiently robust to remain following annealing (Fig. 2b). We tested various organic compounds both computationally and experimentally. We first performed DFT simulations to calculate the binding energy and investigate the energy level alignments by using a hybrid exchange-correlation functional of B3LYP (Methods). We started from organic molecules with a $\mathrm{P}=\mathrm{X}$ end group ( $\mathrm{X}$ is oxygen, sulfur or selenium), such as triphenylphosphine oxide (TPPO), triphenylphosphine sulfide (TPPS) and triphenylphosphine selenide (TPPSe). We found that the $\mathrm{P}=\mathrm{O}: \mathrm{Pb}$ dative bond showed a strongest binding energy of $1.1 \mathrm{eV}$. We also explored other oxides with a $\mathrm{Y}=\mathrm{O}$ end group ( $\mathrm{Y}$ is carbon, nitrogen, sulfur or arsenic), such as nitrosobenzene (PNO), benzophenone (DPCO), diphenyl sulfoxide (DPSO) or triphenylarsine oxide (TPAsO) to compare with TPPO. We found that the $\mathrm{P}=\mathrm{O}: \mathrm{Pb}$ dative bond was also stronger than $\mathrm{S}=\mathrm{O}: \mathrm{Pb}(0.8 \mathrm{eV})$ and $\mathrm{Pb}$ binding with the physically absorbed $\mathrm{O}_{2}(0.3 \mathrm{eV})$. Energy level alignment calculation revealed that PNO and DPCO introduce states that reside within the perovskite bandgap (Fig. 2b). These trends were seen in PLQY studies, which indicated that perovskites edge-stabilized by TPPO and TPAsO showed superior PLQYs of $97 \%$ and $92 \%$, respectively, much higher than films treated with other molecules (with PLQYs from $0.1 \%$ to $40 \%$ ) (Fig. 2c).

Photothermal stability. Photoluminescence (PL) spectra of edgestabilized perovskite films show narrower linewidth compare with control perovskites (Supplementary Fig. 8). The phosphine oxides introduced in situ during the perovskite crystallization process, removed edge state defects and also tightened the distribution of quantum wells, resulting in a narrowband emission and enabling fast energy funneling in cascade energy structure. We then monitored the PL stability of the TPPO-treated perovskites and untreated controls under continuous excitation by using a $374-\mathrm{nm}$ laser diode $\left(8 \mathrm{~mW} \mathrm{~cm}^{-2}\right)$ in air with a relative humidity of $10 \%$ (Fig. 3a). The emission of the untreated controls degraded to $40 \%$ of its initial value within $1 \mathrm{~h}$, and was 
a

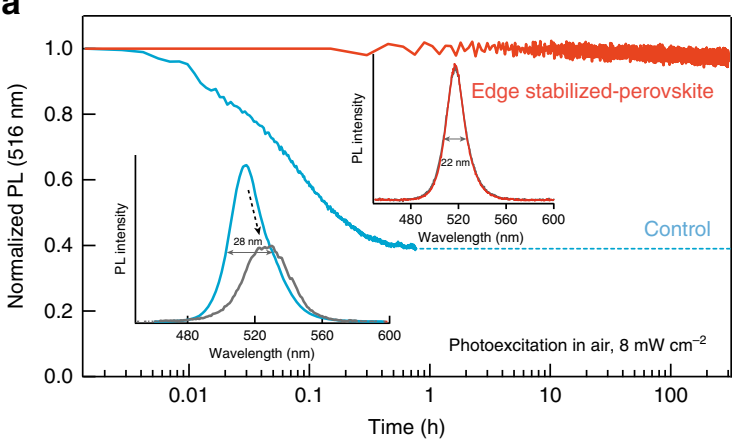

C

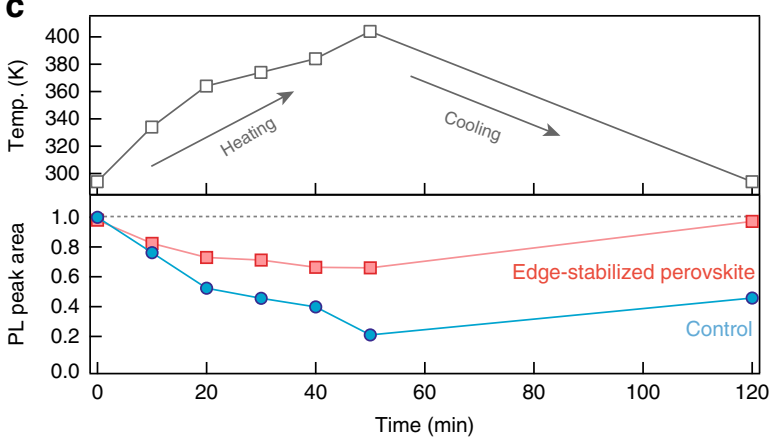

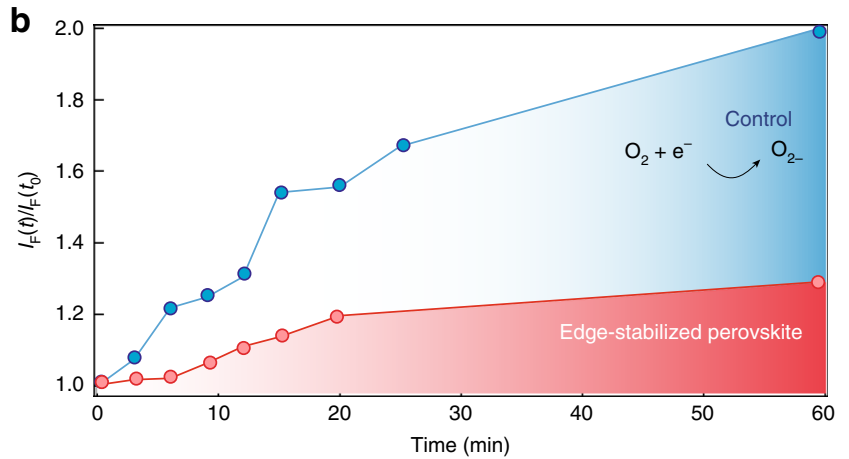

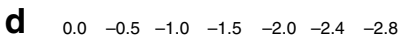
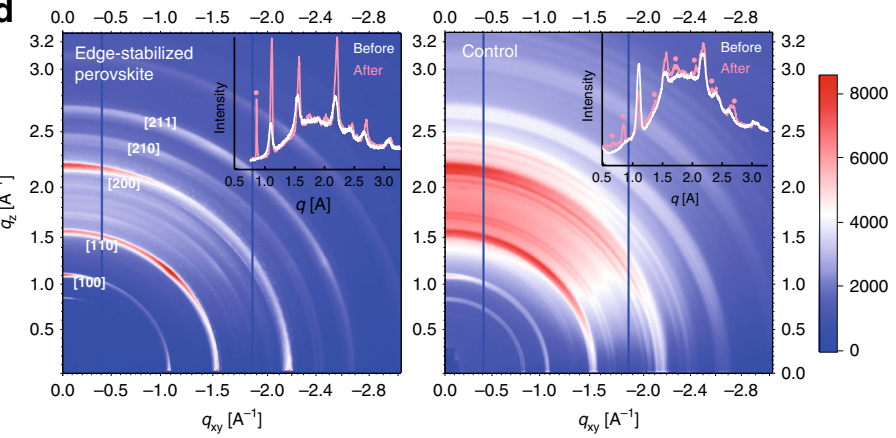

Fig. 3 Photothermal stability. a PL stability under continuous excitation under a 374-nm laser diode. The inset shows PL spectra of the untreated control and edge-stabilized sample before (in red and blue, respectively) and after (in gray) measurement. b Normalized fluorescence intensity of the superoxide probe solution. c Thermal stability of the untreated control and edge-stabilized perovskite under a continuous heat stress. $\mathbf{d}$ In situ GIWAXS of the untreated control and edge-stabilized perovskite. The films were gradually annealed up to $150^{\circ} \mathrm{C}$, let there for 30 min before cooling down and measured. The inset curves show the out-of-plane line profiles before and after heat stress.

accompanied by broadened and red-shifted spectra. By contrast, the TPPO-treated perovskites retained their initial brightness and emission peak position over the course of continuous illumination for $300 \mathrm{~h}$. To ascertain whether superoxide production played a key role in these films under photoexcitation, we measured superoxide generation under illumination. We used a superoxide-sensitive dye as a reporter (Supplementary Fig. 9), placed within the films, and monitored the increase of PL intensity of the dye (peaked at $610 \mathrm{~nm}$ ) associated with the evolution of superoxide (Fig. 3b). The PL intensity of the dye doubled within $1 \mathrm{~h}$ relative to the initial intensity. Here we studied the ratio of $I_{\mathrm{F}}(t) / I_{\mathrm{F}}\left(t_{0}\right)$, where $I_{\mathrm{F}}(t)$ shows the fluorescence intensity at time $t$ and $I_{\mathrm{F}}\left(t_{0}\right)$ indicates the background fluorescence intensity of the probe the superoxide probe dye molecule at $t=0$. This figure reports the yield of superoxide generation, and the result agrees well with the prior reports with bulk perovskites degradation mechanisms. The emissive properties of the edgestabilized perovskites exhibited reversibility following thermal stress, and recovered their near-unity PLQY following heating at $150{ }^{\circ} \mathrm{C}$ (Fig. 3c and Supplementary Fig. 10). In the case of the untreated control, most of the PL intensity was lost during the heating process, and only 50\% was recovered after cooling to room temperature. In addition, in situ grazing incidence wideangle X-ray scattering (GIWAXS) (Fig. 3d and Supplementary Fig. 11) showed that edge-stabilized perovskites kept their initial structural phase and crystallinity following heat stress. In contrast, the untreated control exhibited increased disorder, as evidenced by broader rings, and the appearance of additional peaks associates with structural degradation.

Edge-stabilization mechanism. Next we fabricated single crystals of the reduced-dimensional MHPs with the composition of
$\mathrm{PEA}_{2} \mathrm{CsPb}_{2} \mathrm{Br}_{7}$ (Supplementary Fig. 12) and exfoliated them into few-hundred-micrometer-sized thin flakes to distinguish the edge and the center of the samples using optical microscopy. Figure $4 \mathrm{a}-\mathrm{e}$ shows the mechanically exfoliated $\mathrm{PEA}_{2} \mathrm{CsPb}_{2} \mathrm{Br}_{7}$ $(n=2)$ crystals, which have been reported to exhibit edge states with PL emission from low energy $(520 \mathrm{~nm})$ when exposed to $\operatorname{air}^{31}$. This is assigned to the stochastic loss of PEA and formation of bulk $\mathrm{CsPbBr}_{3}$ perovskites. The PL intensity from edge states increased twofold upon in situ addition of phosphine oxide molecules. We attribute this to the passivation of bulk perovskites located at the crystal edges. The PL from the bulk crystal $(n=2)$ did not change an observation we account for by noting that these crystals were oriented along the $<001>$ direction, and the organic amine molecules were protecting the surface of the crystals ${ }^{19}$, the phosphine oxide molecules were selectively passivate edge state and enhanced the PL. We also employed confocal time-resolved PL decay measurements to verify the enhanced lifetime of edge states when we used phosphine oxides (Supplementary Fig. 13).

To verify that $\mathrm{P}=\mathrm{O}$ bound the perovskites via direct chemical linkages and was not merely incorporated nonspecifically alongside the precursor, we conducted a study that combined Raman spectroscopy, solid-state nuclear magnetic resonance (NMR) spectroscopy, Fourier-transform Infrared spectroscopy (FTIR), X-ray photon spectroscopy (XPS) and X-ray diffraction (XRD) measurements. The Raman spectrum of TPPO agreed with the established literature frequency values, and changed significantly upon addition to the $\mathrm{PbBr}_{2}$ precursor or to the perovskites (Supplementary Figs. 14 and 15). Additionally, we utilized solidstate ${ }^{31} \mathrm{P}$ NMR spectroscopy to investigate the interaction of TPPO with perovskites. We observed chemical shifts of the TPPOprecursor (TPPO- $\mathrm{PbBr}_{2}$ ) and TPPO-perovskite relative to TPPO itself, indicative of changes in the coordination of phosphorus (Fig. 4f $)^{32}$. The narrow NMR peak for the TPPO-perovskite 

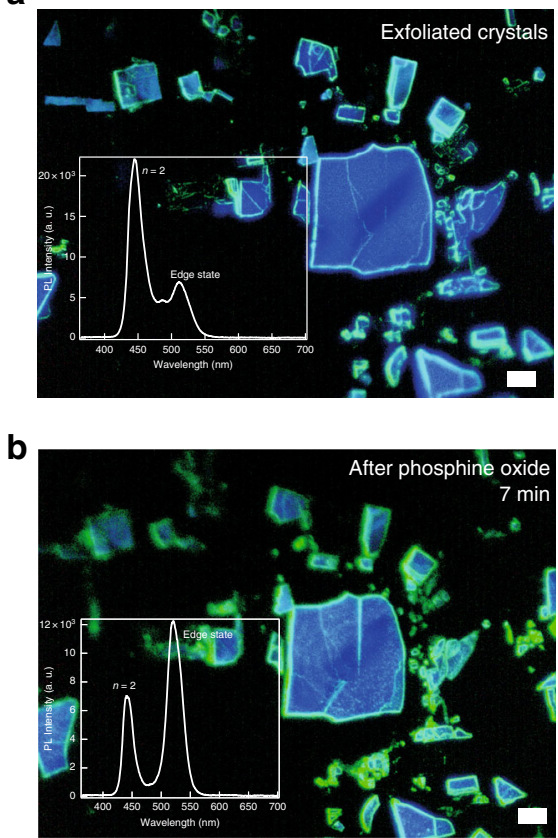

C

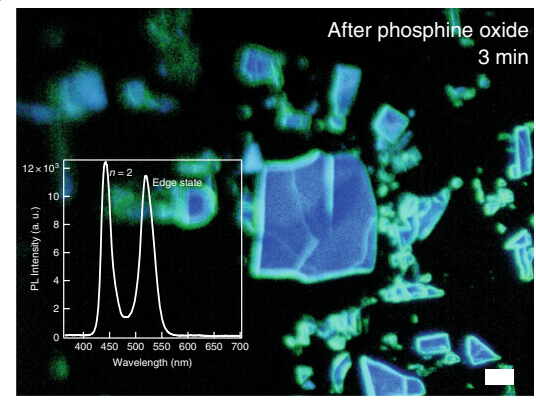

d

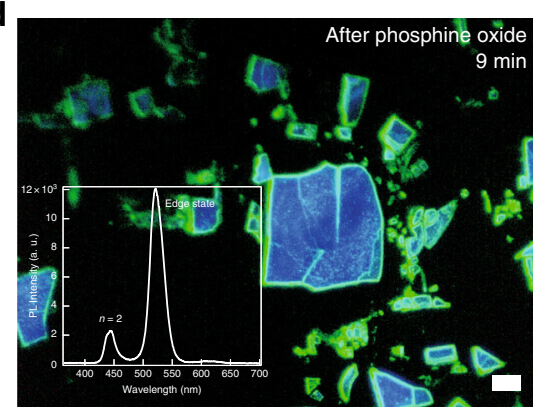

e

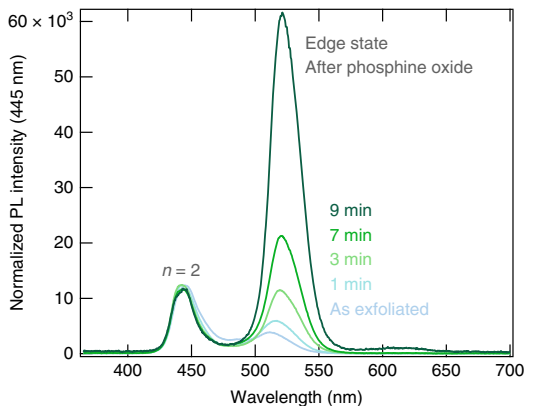

f

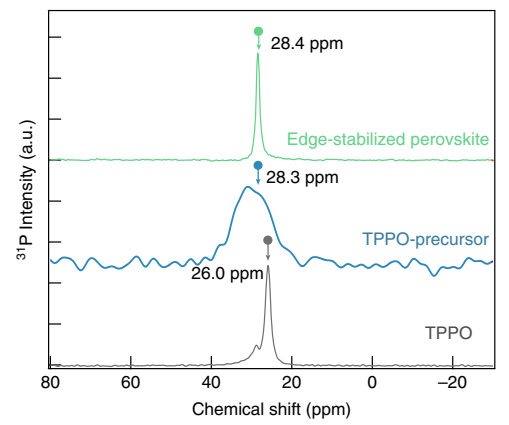

Fig. 4 Origins of edge stabilization. a Microscopic image of the mechanically exfoliated $\mathrm{PEA}_{2} \mathrm{CsPb}_{2} \mathrm{Br}_{7}(n=2)$ single crystal under continuous excitation by using a $374-\mathrm{nm}$ laser diode. The inset shows PL spectra, from the both intrinsic $\left(\lambda_{\mathrm{em}}=450 \mathrm{~nm}\right)$ and edge state $\left(\lambda_{\mathrm{em}}=510 \mathrm{~nm}\right)$ emission. Scale bar is $10 \mu \mathrm{m}$. b-d Microscopic images as function of time after in situ addition of TPPO solution. e Normalized PL spectra extracted from the microscope images. Scale bar is $10 \mu \mathrm{m}$. ${ }^{31} \mathrm{P}$-NMR spectra of TPPO only, TPPO-precursor ( $\mathrm{TPPO}^{-\mathrm{PbBr}_{2}}$ ) and TPPO-perovskites to monitor the interaction between $\mathrm{P}=\mathrm{O}$ and $\mathrm{Pb}$ in perovskites.

sample indicated that TPPO assumed a single configuration in the sample, as opposed to the broad range of structures evident in the TPPO-precursor (TPPO- $\mathrm{PbBr}_{2}$ ) spectrum. We measured FTIR spectra of perovskite with and without TPPO edge stabilization and compared to those of TPPO itself (Supplementary Fig. 16). The stretching vibration of $\mathrm{P}=\mathrm{O}$ in TPPO itself appeared at 1182 $\mathrm{cm}^{-1}$ and was shifted to $1179 \mathrm{~cm}^{-1}$ upon the formation of TPPO$\mathrm{PbBr}_{2}$ in the perovskite edge-stabilized by TPPO. We attribute this to a weakened $\mathrm{P}=\mathrm{O}$ bond caused by the interaction with $\mathrm{Pb}^{2+}$ in the perovskites. In addition to the above observations, the edgestabilized perovskite also showed additional IR absorption at $723 \mathrm{~cm}^{-1}$, indicating the interaction of the phosphine oxide and perovskites $(\mathrm{P}=\mathrm{O}: \mathrm{Pb})$. We also found that TPPO was incorporated into perovskite films during the spin-coating process when delivered using an anti-solvent. We observed two additional XRD peaks in these films, at $2 \theta=10.11^{\circ}$ and $20.22^{\circ}$, corresponding to the diffraction from (TPPO) ${ }_{2} \mathrm{PbBr}_{2}$ complexes (Supplementary Fig. 17 for the XRD of TPPO-precursor reference) ${ }^{33}$. XPS was used to determine the presence of phosphor and oxygen atoms in TPPO-treated perovskites. XPS core-level photoemission spectra of $\mathrm{C} 1 s, \mathrm{~Pb} 4 f, \mathrm{P} 2 p, \mathrm{O} 1 s$ and $\mathrm{Br} 3 d$ are shown in Supplementary Fig. 18. The results reveal the existence of $\mathrm{P}$ in the TPPOprecursor and edge-stabilized perovskites.

Device performance and operational stability. We then sought to translate the bright and stable perovskite films into highefficiency LEDs. We used a device architecture consisting of ITO/PEDOT:PSS:PFI/Perovskite/TPBi/LiF/Al (Fig. 5a, b) (ITO: Indium Tin Oxide; PEDOT:PSS:PFI: poly(3,4-23122-1-122+ -122 ethylenedioxythiophene)polystyrene sulfonate doped with perfluorinated ionomer; LiF: Lithium Fluoride). We used PEDOT:PSS:PFI as the hole transport layer in view of its excitonbuffering and hole-injection capabilities ${ }^{34}$, together with 1,3,5-tris
(N-phenylbenzimiazole-2-yl)benzene (TPBi) as the electron transport layer, and $\mathrm{LiF} / \mathrm{Al}$ as the cathode. Ultraviolet photoemission spectroscopy (UPS) measurements were used to determine the valence band positions and work functions of the control and edge-stabilized perovskites (Fig. 5a and Supplementary Fig. 19). The work function of the samples was obtained from the ultraviolet radiation energy $(21.2 \mathrm{eV})$, and the energies at secondary cut-offs of UPS spectra. Valence band ionization energy (IE) decreased from 6.55 to $6.14 \mathrm{eV}$ in the edge-stabilized perovskites, due to the electronic structure change caused by surface modification. This enables a reduction in the injection barrier of electrons and holes within the devices. We then measured electron and hole only devices to evaluate the charge injection balance in devices. The results showed that in devices, the edge-stabilized perovskites exhibited a higher balance in electron and hole transport than the untreated control (Supplementary Fig. 20). Also, the electroluminescence (EL) spectra did not change in either cases (Supplementary Fig. 21).

We selected TPPO and TPAsO to fabricate the perovskite layer. We found that compared with the untreated control, the perovskites treated with TPPO or TPAsO showed significantly improved EL performance (Fig. 5c, d and Table 1). The perovskite treated with TPPO exhibited a maximum EQE of $14 \%$ and luminance of $4.5 \times 10^{4} \mathrm{~cd} \mathrm{~m}^{-2}$ (corresponding to an EQE 5\%) (Supplementary Figs. 22-24), exceeding the one treated with TPAsO with an EQE of $9 \%$ and maximum luminance of $2.0 \times$ $10^{4} \mathrm{~cd} \mathrm{~m}^{-2}$ (corresponding to an EQE of 2\%). In addition, the current density-voltage-luminance $(J-V-L)$ measurements at various scan rates and directions attest to an absence of hysteresis (Supplementary Figs. 25, 26). Perovskites were protected by PEA ligands and also by the TPPO as edge ligands, and these may help to slow ion migration.

Since the physically absorbed oxygen inside the perovskites contributes to severe photoelectric degradation even in encapsulated 

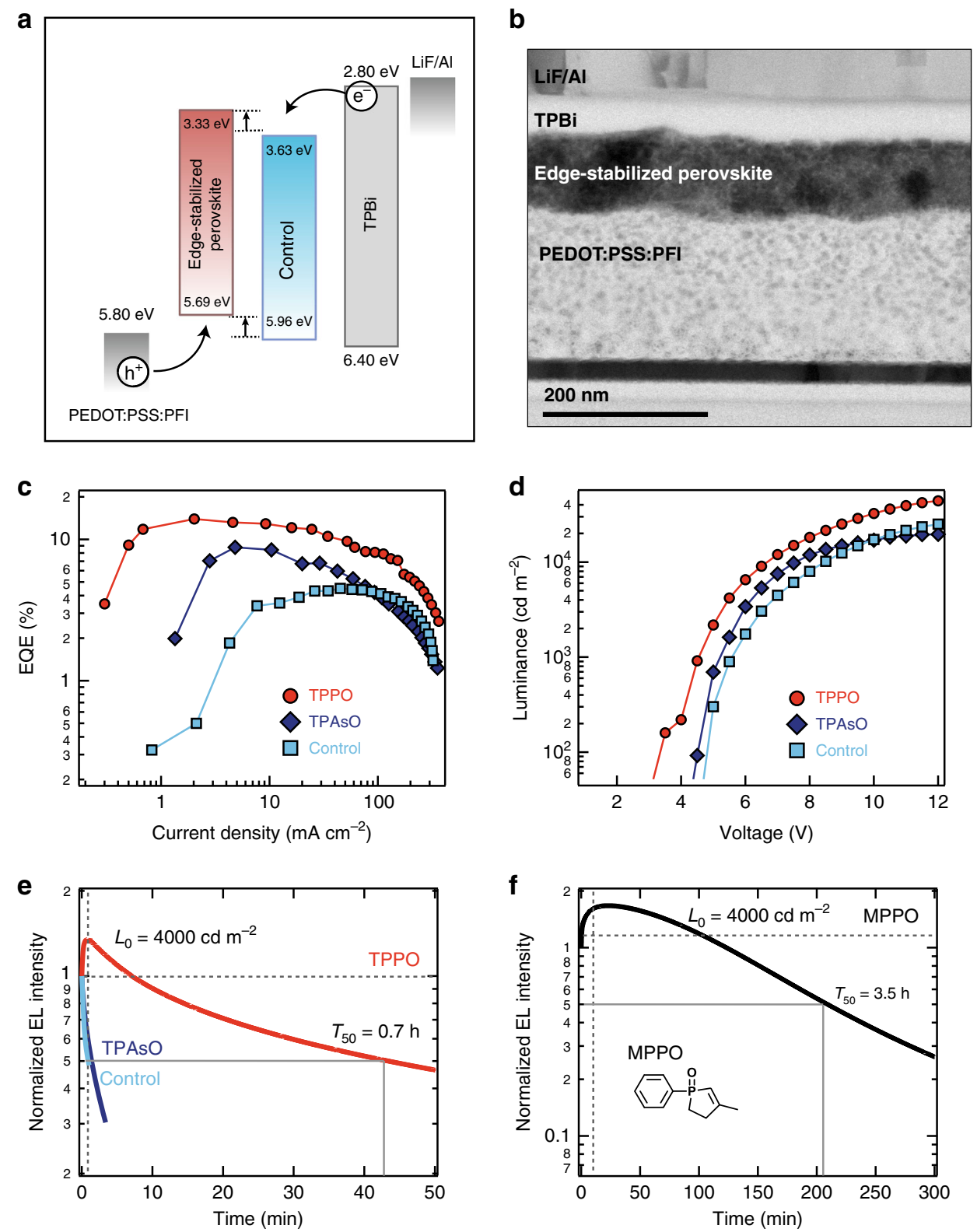

Fig. 5 LED performance and operational stability. a Cross-section focused ion beam (FIB) transmission electron microscope (TEM) image. $\mathbf{b}$ Energy band diagram based on literature and UPS measurements. $\mathbf{c}$ EQE versus current density and $\mathbf{d}$ luminance versus voltage characteristics of untreated controls and edge-stabilized perovskite LEDs. e Operational device stability of untreated controls and edge-stabilized perovskite LEDs at a starting luminance of $4000 \mathrm{~cd} \mathrm{~m}^{-2}$. f Device operational stability of the perovskite LED with MPPO at a starting luminance of $4000 \mathrm{~cd} \mathrm{~m}^{-2}$.

Table 1 Device performance and operational stability of LEDs based on perovskites with and without edge stabilization.

\begin{tabular}{|c|c|c|c|c|c|c|}
\hline Perovskites & PLQY (\%) & $V_{\text {on }}(\mathrm{V})$ & Max EQE (\%) & $\operatorname{Max} L\left(c d m^{-2}\right)$ & $T_{50}$ at $4000 \mathrm{~cd} \mathrm{~m}^{-2}$ & $T_{50}$ at $100 \mathrm{~cd} \mathrm{~m}^{-2}$ \\
\hline TPAsO & 92 & 4.5 & 8.8 & 19,990 & $82 \mathrm{~s}$ & $12 \mathrm{~min}$ \\
\hline TPPO & 98 & 3.5 & 14.0 & 45,230 & $44 \mathrm{~min}$ & $33 \mathrm{~h}$ \\
\hline
\end{tabular}

devices, operational instability remains a critical issue in perovskite LEDs $^{9}$. We therefore explored whether the edge-stabilization strategy could enhance device operational stability. The LEDs were biased to achieve an initial luminance of $4000 \mathrm{~cd} \mathrm{~m}^{-2}$; we then studied the variation in their EL intensity (Fig. 5e). Control perovskites with no edge stabilization, as well as the perovskites treated with TPAsO, lost $50 \%$ of the initial emission within 53 and $82 \mathrm{~s}$, respectively, while the perovskite treated with TPPO showed a longer half-lifetime $\left(T_{50}\right)$ of $44 \mathrm{~min}$. Similar stability trends were also observed at a lower initial luminance of $100 \mathrm{~cd} \mathrm{~m}^{-2}$ (Table 1). We therefore designed another molecule, 3-methyl-1phenyl-2-phospholene 1-oxide (MPPO), which had a smaller steric hindrance, and could bind $\mathrm{Pb}$ more effectively. We achieved a much longer $T_{50}$ of $3.5 \mathrm{~h}$ at $4000 \mathrm{~cd} \mathrm{~m}^{-2}$ (Fig. 5f and Supplementary Fig. 27). Comparing among all the previous reports, including those that reported lifetimes only at low luminance, this work provides 
the highest brightness ever observed in a long-lived LED (initial luminance of $3800 \mathrm{~cd} \mathrm{~m}^{-2}$, with $\left.T_{50}=10 \mathrm{~min}\right)^{20}$. Moreover, we attribute a sharp degradation of the device after $3.5 \mathrm{~h}$ operation to the interface-induced chemical reaction between the perovskite and charge transport layer that accelerates the materials degradation rather than to perovskite degradation itself. The interfacial contact between perovskite/TPBi and $\mathrm{LiF} / \mathrm{Al}$ has been reported to be another critical factor limiting operational stability. The edgestabilized perovskites retained the PL intensity in air over $300 \mathrm{~min}$ on ITO/ZnO/PVP, but exhibited faster decay (lose $25 \%$ initial PL in 5 min) on PEDOT:PSS:PFI layers (Supplementary Fig. 28). The acidic nature of PEDOT:PSS caused corrosion of the active materials, highlighting the importance of further device interface engineering to improve stability.

\section{Discussion}

In summary, we demonstrate an edge-stabilization strategy that achieves bright and stable reduced-dimensional perovskites with high PLQYs and suppressed photodegradation. We incorporate phosphine oxides during film fabrication and then passivate otherwise exposed layer edges. The resulting perovskites exhibit a remarkable robustness against oxygen, moisture and heat. When implemented as active layers in LEDs, they showed a peak EQE of $14 \%$, maximum luminance of $4.5 \times 10^{4} \mathrm{~cd} \mathrm{~m}^{-2}$, and an operational half-lifetime of $3.5 \mathrm{~h}$ at $4000 \mathrm{~cd} \mathrm{~m}^{-2}$ under continuous operation. This is 21 times longer than the best green LEDs previously reported. Our edge-stabilization strategy can be applied to other types of perovskites, including quantum dots and polycrystalline films with a range of emission wavelengths.

\section{Methods}

Fabrication of perovskite films. In $\mathrm{PEA}_{2} \mathrm{Cs}_{2.4} \mathrm{MA}_{0.6} \mathrm{~Pb}_{4} \mathrm{Br}_{13}$ perovskite, precursors $\mathrm{PbBr}_{2}$ (0.6 M) (99.999\% Alfa-Aesar), CsBr (0.36 M) (99.999\%, Sigma-Aldrich), $\operatorname{MABr}(0.1 \mathrm{M})$ (Dyesol) and PEABr $(0.3 \mathrm{M})$ (Dyesol) were dissolved in DMSO. The precursor was spin-coated onto a glass substrate using a two-step method ${ }^{35}$. During the second step of the spin-coating process, $100-500 \mu \mathrm{L}$ of chloroform was dropped onto the substrate. For the edge-stabilized perovskite films, TPPO ( $98 \%$, Sigma-Aldrich) was dissolved in chloroform (5-10 $\mathrm{mg} \mathrm{mL}^{-1}$ ) and deposited onto the perovskite film during the second step. The resulting films were then annealed at $90{ }^{\circ} \mathrm{C}$ for $7 \mathrm{~min}$ to increase crystallization.

\section{Device fabrication and characterization. A mixed solution of PEDOT:PSS} (Clevios ${ }^{\mathrm{TM}}$ PVP Al4083) and perfluorinated ionomer, tetrafluoroethylene-perfluoro-3,6-dioxa-4-methyl-7-octenesulfonic acid copolymer (PFI) (PEDOT:PSS: PFI $=1: 6: 25.4$ (mass ratio)), was spin-coated on the ITO-coated glass substrates treated with oxygen-plasma; and this was followed by an annealing step on a hot plate at $150{ }^{\circ} \mathrm{C}$ for $20 \mathrm{~min}$ in air $^{36}$. Perovskite precursor solutions were spin-coated onto the PEDOT:PSS-coated substrate via the two-step anti-solvent spin-coating method. TPBi $(60 \mathrm{~nm})$ and $\mathrm{LiF} / \mathrm{Al}$ electrodes $(1 \mathrm{~nm} / 100 \mathrm{~nm})$ were deposited using a thermal evaporation system under a high vacuum of $<10^{-4} \mathrm{~Pa}$. The device active area was $6.14 \mathrm{~mm}^{2}$ as determined by the overlap between the ITO and Al electrodes. LEDs were encapsulated prior to measurement. All devices were tested in ambient conditions (Supplementary Fig. 30)

We developed an experimental setup for organic LED measurements based on a procedure adapted from Forrest et al. ${ }^{37}$. The angular dependence of intensity and EL spectrum are taken into consideration via the use of the integrating sphere, which collects emitted light across angles. The current density-voltage $(J-V)$ characteristic was measured using a Keithley 6430 source meter. The absolute radiation flux for calculating the EQE, power efficiency and luminance was collected with a measurement system containing an integrating sphere and an Ocean Optics USB4000 spectrometer, which was calibrated with a standard halogen lamp (Ocean Optics HL-2000). The devices were mounted on the open aperture of the integrating sphere to allow the light emitted from the glass surface to be collected; while the emission from the substrate edges was not collected. When calculating the luminance, a Lambertian emission profile was assumed (Supplementary Fig. 31). The integration time for the spectrometer was set for $10 \mathrm{~ms}$ in order not to saturate the CCD detector during measurement. We have reproduced the measurements multiple times and also for several repetitions of the same experiment. The device was encapsulated for the efficiency measurement with a UV-resin (exposure under UV light for $10 \mathrm{~s}$ ) and covered on the device and a transparent glass substrate.

We used half-lifetime $\left(T_{50}\right)$, the time after which the device luminance drops to $50 \%$ of the initial value, to describe the device operational stability. The half-lifetime measurements were carried out in a nitrogen-filled glovebox without encapsulation. Devices were driven by a Keithley 2400 source meter at constant current, and the luminance intensity was measured using a commercial photodiode (Vishay Semiconductors BPW34). The current density used to drive the LEDs was first determined using the EQE measurement system. To achieve a high initial luminance of $4000 \mathrm{~cd} \mathrm{~m}^{-2}$, the current densities were: control devices: $25 \mathrm{~mA} \mathrm{~cm}^{-2}$; TPAsOtreated: $15 \mathrm{~mA} \mathrm{~cm}^{-2}$; and TPPO-treated $8 \mathrm{~mA} \mathrm{~cm}^{-2}$. To achieve an initial luminance of $100 \mathrm{~cd} \mathrm{~m}^{-2}$, the current density for the LEDs were: control $2.5 \mathrm{~mA} \mathrm{~cm}^{-2}$ and TPAsO $1.5 \mathrm{~mA} \mathrm{~cm}^{-2}$. For the LEDs based on MPPO, we used $10 \mathrm{~mA} \mathrm{~cm}^{-2}$ to achieve an initial luminance of $4000 \mathrm{~cd} \mathrm{~m}^{-2}$. The photodiode was biased at $0 \mathrm{~V}$ and the photocurrent, proportional to the luminance, was recorded at $2 \mathrm{~s}$ intervals.

Photoluminescence measurement. A Horiba Fluorolog system was used for PL measurements. Steady-state PL was measured with a monochromatized Xe lamp as the excitation source. A Time Correlated Single Photon Counting detector and a pulsed UV laser diode $(\lambda=374 \mathrm{~nm})$ were used to acquire transient PL. An instrument response function of $\Delta t \sim 0.13 \mathrm{~ns}$ limits the overall time resolution. Time-resolved emission spectra were recorded by measuring individual transient PL traces at increasing emission wavelengths. PLQYs were obtained by coupling a Quanta-Phi integrating sphere to the Fluorolog system through optical fiber bundles. All PLQY measurements followed published methods ${ }^{38}$. We measured the both excitation and emission spectra, where the sample was directly excited by the excitation beam path in the integrating sphere, the sample offset within the integrating sphere from the beam path; and we also measured the empty sphere itself in order to acquire information regarding the excitation beam. We include raw spectra of near-unity PLQY data from edge-stabilized perovskite in Supplementary Fig. 32. The PL spectrum of perovskite controls and edge-stabilized perovskites reveal emission centered at $517 \mathrm{~nm}$, with the edge-stabilized perovskite films showing full-width at half-maximum of $22 \mathrm{~nm}$ compared to $28 \mathrm{~nm}$ for the control To investigate the role of TPPO on the passivation of edge traps, we carried out temperature-dependent PL measurements. As the temperature decreases, the PL intensity of unpassivated perovskites steadily increases, indicative of trap freezing that results in reduced trap recombination losses. The PL intensity of the edgestabilized perovskites, on the other hand, remains unchanged, suggesting negligible trapping even at room temperature. This result agrees with the measured nearunity PLQY values of edge-stabilized perovskites $(97 \pm 2 \%)$ compared to the case of control perovskite samples $(60 \pm 10 \%)$ and the radiative decay time of edgestabilized perovskites (Supplementary Fig. 33). The PL stability test was performed with a nanosecond pulsed diode laser, with continuous $374 \mathrm{~nm}$ excitation at $1 \mathrm{~mW}$ $\mathrm{cm}^{-2}$ power density. All perovskite films were tested in air with relatively low humidity. The photothermal stability test was performed by placing the sample on an aluminum block with a resistive heater. A thermocouple and temperature controller were used to maintain active feedback. The sample was excited using a $442 \mathrm{~nm}$ diode laser. The light was then coupled to a spectrometer (USB200+).

Transmission electron microscopy. High-resolution HAADF-STEM images were acquired using a probe-corrected FEI Titan microscope operating at $300 \mathrm{kV}$. A probe semiconvergence angle of $\sim 20 \mathrm{mrad}$ was used. Due to the sensitivity of layered perovskites to the electron beam, a reduced dose below $10 \mathrm{e} \mathrm{A}^{-2} \mathrm{~s}^{-1}$ was used during the imaging.

Device cross-section focused ion beam TEM. Transmission electron microscopy (TEM) samples were prepared using an FEI Helios NanoLab 400S focused ion beam (FIB)/SEM dual-beam system equipped with a $\mathrm{Ga}+$ ion source. $\mathrm{C} / \mathrm{Pt}$ layers were deposited on the surface region of interested via electron and ion beam for protection. The sample was thinned to $80 \mathrm{~nm}$ lamella using progressively decreasing ion beam energies in the FIB down to $2 \mathrm{keV}$.

GIWAXS measurements. GIWAXS measurements were performed at beamline 7.3.3 at the Advanced Light Source, Lawrence Berkeley National Laboratory. The wavelength of the X-ray beam employed was $1.24 \AA$. The scattering patterns were obtained at a photon incidence angle of $0.25^{\circ}$ with respect to the sample plane. Samples were scanned in a $\mathrm{He}$ environment to reduce air scattering. Exposure times were typically between $5 \mathrm{~s}$ and $30 \mathrm{~s}$. The scattering patterns were recorded using a Pilatus $2 \mathrm{M}$ detector at a fixed distance of $277.674 \mathrm{~mm}$. Calibration of the lengths in reciprocal space was carried out by using silver behenate. A heating stage was set up for temperature-dependent in situ studies. The GIWAXS scans were taken from $90^{\circ} \mathrm{C}$ to $150^{\circ} \mathrm{C}$. The annealing temperature was increased by $10^{\circ} \mathrm{C}$ at a time, and kept at $150^{\circ} \mathrm{C}$ for $30 \mathrm{~min}$. After the temperature elevating process, the samples were cooled down to $50^{\circ} \mathrm{C}$. Samples for GIWAXS were spin-coated on glass substrates following the same spin coating and annealing procedures as were used in fabricating LEDs.

31P NMR measurements. The samples were finely ground, then packed evenly into $4 \mathrm{~mm}$ zirconia rotor, and sealed at the open end with a Vespel cap. The ${ }^{31} \mathrm{P}$ NMR spectra were acquired using a Bruker $400 \mathrm{MHz}$ AVANACIII NMR spectrometer equipped with double resonance Bruker MAS probe (BrukerBioSpin, Rheinstetten, Germany). The spectra were recorded using onepulse pulse program with $14 \mathrm{kHz}$ spinning rate. To achieve high signal to noise ratio, the spectra were 
recorded by collecting at least $1 \mathrm{k}$ scans with recycle delay time of $10 \mathrm{~s}$. Bruker Topspin 3.2 software (Bruker BioSpin, Rheinstetten, Germany) was used to record the NMR spectra and to analyze the data.

${ }^{31} \mathrm{P}$ NMR was used to study of the tri-octylphosphine chalconide moieties capping the surface of CdSe nanocrystals. We observed solid-state ${ }^{31} \mathrm{P}$ NMR chemical shifts in TPPO-perovskite compared to bare TPPO, indicative of changes in the coordination of phosphorus ${ }^{39}$. The narrow NMR peak for the edge-stabilized perovskite sample indicates that TPPO assumes a single configuration in the sample, in contrast to the broad range of structures evident in the TPPO-precursor spectrum. The weakening of the $\mathrm{P}=\mathrm{O}$ bond signal upon the coordinating to the metal surface presented in ${ }^{31} \mathrm{P}$ NMR measurement. The increase of chemical shift in TPPO-perovskite has been observed from a decrease in the electron density at phosphorous due to the oxygen coordination with $\mathrm{Pb}$. The signal in NMR spectroscopy can also depend on the crystal facet to which the element is adsorbed. A peak broadening in TPPO-precursor sample would suggest the presence of species on different surfaces.

DFT simulations. Calculations were performed using the Quickstep module of the CP2K computational package ${ }^{40}$, using a MOLOPT double-zeta plus polarized orbital basis set ${ }^{31}$, Goedecker-Teter-Hutter pseudopotentials ${ }^{32}$, grid cut-off of $600 \mathrm{Ry}$, and Perdew-Burke-Ernzerhof exchange-correlation functional ${ }^{33}$. Layered lead bromide perovskites with $n=3$ were modeled using Cs as a cation both inside and on the surface for computational efficiency. To represent better the bandgaps and level alignments of the molecules with perovskite, free molecules were computed using the B3LYP functional, using a $\mathrm{Cl}_{2}$ molecule as a common energy reference level. The results for the molecule + perovskite calculated at the PBE level are consistent with the findings from B3LYP for free molecules ${ }^{34}$.

A supercell made of $4 \times 3 \times 3$ orthorhombic (Pnma) unit cells was constructed, with only $\Gamma$ k-point used for simulations. The slabs are separated by $30 \AA$ of vacuum in the $z$-direction, made periodic in the $x$-direction, and expose unpassivated edges along the $y$-direction, with $20 \AA$ between the periodic images. All geometries were relaxed until forces on atoms converged to below $40 \mathrm{meV}^{-1}$, including the cell-size degrees of freedom.

The edges in the $y$-direction are cut along the (110) direction of the orthorhombic cell (corresponding to (100) direction in cubic notation), in agreement with TEM images of the $\mathrm{CsPbBr}_{3}$ nanoplatelets and colloidal nanocrystals ${ }^{41,42}$. The edges are saturated by $\mathrm{Cs} \mathrm{Br}$ (or PEA Br) and do not expose any $\mathrm{Pb}$. However, slabs prepared in such a way have an overall excess of cations, which leads to either charging or the electronic doping of such systems which in turn become prone to the desorption of excess cations (Cs or PEA). The first candidate for desorption is Cs at the corners, but the charge balance requires even more cations to be desorbed, leading to openings along the edges. One can also expect that a desorption of a charge-neutral $\mathrm{CsBr}$ or PEABr can be possible, especially near the already exposed site with one ligand lost, and as a result exposing one more $\mathrm{Pb}$. All three types of defects expose one dangling bond of $\mathrm{Pb}$, which is susceptible to molecular adsorption. DMSO, TPPO and $\mathrm{O}_{2}$ molecules were adsorbed onto the remaining exposed $\mathrm{Pb}$ dangling bond. Binding energies were calculated as a difference between $E_{\text {surf+molecule }}, E_{\text {surf }}$ and $E_{\text {molecule_in_gas_phase. }}$ Entropy effects were not computed as they typically do not exceed $0.1 \mathrm{eV}$.

\section{Data availability}

The data that support the findings of this study are available from the corresponding author upon reasonable request.

Received: 27 May 2019; Accepted: 9 December 2019; Published online: 10 January 2020

\section{References}

1. Xiao, Z. G. et al. Efficient perovskite light-emitting diodes featuring nanometre-sized crystallites. Nat. Photonics 11, 108-115 (2017).

2. Quan, L. N. et al. Ligand-stabilized reduced-dimensionality perovskites. J. Am. Chem. Soc. 138, 2649-2655 (2016).

3. Tsai, H. et al. High-efficiency two-dimensional Ruddlesden-Popper perovskite solar cells. Nature 536, 312-316 (2016)

4. Tan, Z. K. et al. Bright light-emitting diodes based on organometal halide perovskite. Nat. Nanotechnol. 9, 687-692 (2014).

5. Jeon, T. et al. Hybrid perovskites: effective crystal growth for optoelectronic applications. Adv. Energy Mater. 7, 1602596 (2017).

6. Quan, L. N., Garcia de Arquer, F. P., Sabatini, R. P. \& Sargent, E. H. Perovskites for light emission. Adv. Mater. 30, e1801996 (2018).

7. Quan, L. N. et al. Perovskites for next-generation optical sources. Chem. Rev. 119, 7444-7477 (2019).

8. Cohen, B. E., Wierzbowska, M. \& Etgar, L. High efficiency and high open circuit voltage in quasi 2D perovskite based solar cells. Adv. Funct. Mater. 27, 1604733 (2016).
9. Liao, Y. et al. Highly oriented low-dimensional tin halide perovskites with enhanced stability and photovoltaic performance. J. Am. Chem. Soc. 139, 6693-6699 (2017)

10. Tsai, H. et al. High-efficiency two-dimensional Ruddlesden-Popper perovskite solar cells. Nature 536, 312-316 (2016)

11. Cao, D. H., Stoumpos, C. C., Farha, O. K., Hupp, J. T. \& Kanatzidis, M. G. 2D homologous perovskites as light-absorbing materials for solar cell applications. J. Am. Chem. Soc. 137, 7843-7850 (2015).

12. Smith, I. C., Hoke, E. T., Solis-Ibarra, D., McGehee, M. D. \& Karunadasa, H. I. A layered hybrid perovskite solar-cell absorber with enhanced moisture stability. Angew. Chem. Int. Ed. 53, 11232-11235 (2014).

13. Pedesseau, L. et al. Advances and promises of layered halide hybrid perovskite semiconductors. ACS Nano 10, 9776-9786 (2016).

14. Mao, L. et al. Tunable white-light emission in single-cation-templated threelayered 2D perovskites (CH3CH2NH3)4Pb3Br10-x Clx. J. Am. Chem. Soc. 139, 11956-11963 (2017).

15. Quan, L. N. et al. Tailoring the energy landscape in quasi-2D halide perovskites enables efficient green-light emission. Nano Lett. 17, 3701-3709 (2017).

16. Yuan, M. et al. Perovskite energy funnels for efficient light-emitting diodes. Nat. Nanotechnol. 11, 872-877 (2016).

17. Zhang, L. et al. Ultra-bright and highly efficient inorganic based perovskite light-emitting diodes. Nat. Commun. 8, 15640 (2017).

18. Kovalenko, M. V., Protesescu, L. \& Bodnarchuk, M. I. Properties and potential optoelectronic applications of lead halide perovskite nanocrystals. Science 358, 745-750 (2017)

19. Shi, E. Z. et al. Extrinsic and dynamic edge states of two-dimensional lead halide perovskites. ACS Nano 13, 1635-1644 (2019).

20. Lin, K. et al. Perovskite light-emitting diodes with external quantum efficiency exceeding 20 per cent. Nature 562, 245-248 (2018).

21. Aristidou, N. et al. Fast oxygen diffusion and iodide defects mediate oxygeninduced degradation of perovskite solar cells. Nat. Commun. 8, 15218 (2017).

22. Voznyy, O. et al. A charge-orbital balance picture of doping in colloidal quantum dot solids. ACS Nano 6, 8448-8455 (2012).

23. Brinck, S. \& Infante, I. Surface termination, morphology, and bright photoluminescence of cesium lead halide perovskite nanocrystals. ACS Energy Lett. 1, 1266-1272 (2016)

24. Pashley, M. D. Electron counting model and its spplication to island structures on molecular-beam epitaxy grown $\mathrm{GaAs}(001)$ and $\mathrm{ZnSe}(001)$. Phys. Rev. B 40, 10481-10487 (1989).

25. Zhang, Y. et al. Molecular oxygen induced in-gap states in $\mathrm{PbS}$ quantum dots. ACS Nano 9, 10445-10452 (2015).

26. Lorenzon, M. et al. Role of nonradiative defects and environmental oxygen on exciton recombination processes in $\mathrm{Cs} \mathrm{PbBr} 3$ perovskite nanocrystals. Nano Lett. 17, 3844-3853 (2017).

27. Yang, W. S. et al. High-performance photovoltaic perovskite layers fabricated through intramolecular exchange. Science 348, 1234-1237 (2015).

28. Ahn, N. et al. Highly reproducible perovskite solar cells with average efficiency of $18.3 \%$ and best efficiency of $19.7 \%$ fabricated via Lewis base adduct of lead (II) iodide. J. Am. Chem. Soc. 137, 8696-8699 (2015).

29. Lee, J.-W., Kim, H.-S. \& Park, N.-G. Lewis acid-base adduct approach for high efficiency perovskite solar cells. Acc. Chem. Res. 49, 311 (2016).

30. Wakamiya, A. et al. Reproducible fabrication of efficient perovskite-based solar cells: X-ray crystallographic studies on the formation of $\mathrm{CH} 3 \mathrm{NH} 3 \mathrm{PbI} 3$ layers. Chem. Lett. 43, 711-713 (2014).

31. VandeVondele, J. \& Hutter, J. Gaussian basis sets for accurate calculations on molecular systems in gas and condensed phases. J. Chem. Phys. 127, 114105 (2007).

32. Goedecker, S., Teter, M. \& Hutter, J. Separable dual-space Gaussian pseudopotentials. Phys. Rev. B 54, 1703-1710 (1996).

33. Perdew, J. P., Burke, K. \& Ernzerhof, M. Generalized gradient approximation made simple. Phys. Rev. Lett. 77, 3865-3868 (1996).

34. Grimme, S., Antony, J., Ehrlich, S. \& Krieg, H. A consistent and accurate ab initio parametrization of density functional dispersion correction (DFT-D) for the 94 elements H-Pu. J. Chem. Phys. 132, 154104 (2010).

35. Jeon, N. J. et al. Solvent engineering for high-performance inorganic-organic hybrid perovskite solar cells. Nat. Mater. 13, 897-903 (2014).

36. Cho, $\mathrm{H}$. et al. Overcoming the electroluminescence efficiency limitations of perovskite light-emitting diodes. Science 350, 1222-1225 (2015).

37. Forrest, S. R., Bradley, D. D. \& Thompson, M. E. Measuring the efficiency of organic light-emitting devices. Adv. Mater. 15, 1043-1048 (2003).

38. de Mello, J., Wittmann, H. F. \& Friend, R. H. An improved experimental determination of external photoluminescence quantum efficiency. Adv. Mater. 9, 230-232 (1997)

39. Becerra, L. R., Murray, C. B., Griffin, R. G. \& Bawendi, M. G. Investigation of the surface-morphology of capped Cdse nanocrystallites by P-31 nuclearmagnetic-resonance. J. Chem. Phys. 100, 3297-3300 (1994). 
40. VandeVondele, J. et al. QUICKSTEP: fast and accurate density functional calculations using a mixed Gaussian and plane waves approach. Comput. Phys. Commun. 167, 103-128 (2005).

41. Protesescu, L. et al. Nanocrystals of cesium lead halide perovskites (CsPbX3, $\mathrm{X}=\mathrm{Cl}, \mathrm{Br}$, and $\mathrm{I})$ : novel optoelectronic materials showing bright emission with wide color gamut. Nano Lett. 15, 3692-3696 (2015).

42. ten Brinck, S. \& Infante, I. Surface termination, morphology, and bright photoluminescence of cesium lead halide perovskite nanocrystals. ACS Energy Lett. 1, 1266-1272 (2016).

\section{Acknowledgements}

This publication is based in part on work supported by an award (KUS-11-009-21) from the King Abdullah University of Science and Technology (KAUST), by the Ontario Research Fund Research Excellence Program, by the Ontario Research Fund (ORF), by the Natural Sciences and Engineering Research Council (NSERC) of Canada, and by the US Department of Navy, Office of Naval Research (Grant Award No. N00014-17-12524). H.Y. acknowledges the Research Foundation-Flanders (FWO Vlaanderen) for a postdoctoral fellowship. E.B. gratefully acknowledges financial support by the Research Foundation-Flanders (FWO Vlaanderen). S.B. acknowledges financial support from European Research Council (ERC Starting Grant \#815128-REALNANO). M.B.J.R. and J.H. acknowledge the Research Foundation-Flanders (FWO, Grants G.0962.13, G.0B39.15, AKUL/11/14 and G0H6316N), KU Leuven Research Fund (C14/15/053) and the European Research Council under the European Union's Seventh Framework Programme (FP/2007-2013)/ERC Grant Agreement No. [307523], ERC-Stg LIGHT to M.B.J.R. DFT calculations were performed on the IBM BlueGene Q supercomputer with support from the Southern Ontario Smart Computing Innovation Platform (SOSCIP). M.I.S. acknowledges the Banting Postdoctoral Fellowship program from the Natural Sciences and Engineering Research Council of Canada (NSERC). H.T. acknowledges the Netherlands Organisation for Scientific Research (NWO) for a Rubicon grant (680-50-1511).

\section{Author contributions}

L.N.Q. conceived the study and developed edge-stabilized perovskites, fabricated lightemitting devices and performed stability tests. D.M. assisted material design and perovskite fabrication. D.M., Y.Z., L.G. and J.T. assisted device fabrication, measurements and stability tests. E.B. and S.B. performed TEM measurements. O.V. performed DFT calculations. L.N Q, H.Y., J.H. and M.B.J.R. performed confocal PL microscopy measurements. R.S., Z.P. and D.M. performed Raman spectroscopy measurements. H.T. and M.L. carried out synchrotron x-ray diffraction measurements. P.T., R.Q.B., G.W., J.Z.F. and Y.L. performed XRD, XPS, AFM, FT-IR and UPS measurements. M.I.S. assisted in superoxide generation rate measurements. J.P., O.M., A.A.H.E., D.H.A. and N.W. performed device cross-section TEM and solid-state NMR measurements. O.V., F.P.G.A. and E.H.S. supervised the project. All authors discussed the results and assisted in the preparation of the manuscript.

\section{Competing interests}

The authors declare no competing interests.

\section{Additional information}

Supplementary information is available for this paper at https://doi.org/10.1038/s41467 019-13944-2.

Correspondence and requests for materials should be addressed to Z.-H.L. or E.H.S

Peer review information Nature Communications thanks Wanyi Nie, Haibo Zeng and the other, anonymous, reviewer(s) for their contribution to the peer review of this work.

Reprints and permission information is available at http://www.nature.com/reprints

Publisher's note Springer Nature remains neutral with regard to jurisdictional claims in published maps and institutional affiliations.

(c) (i) Open Access This article is licensed under a Creative Commons Attribution 4.0 International License, which permits use, sharing, adaptation, distribution and reproduction in any medium or format, as long as you give appropriate credit to the original author(s) and the source, provide a link to the Creative Commons license, and indicate if changes were made. The images or other third party material in this article are included in the article's Creative Commons license, unless indicated otherwise in a credit line to the material. If material is not included in the article's Creative Commons license and your intended use is not permitted by statutory regulation or exceeds the permitted use, you will need to obtain permission directly from the copyright holder. To view a copy of this license, visit http://creativecommons.org/ licenses/by/4.0/.

(c) The Author(s) 2020 